\title{
High Altitude Launch for a Practical SSTO
}

\author{
Geoffrey A. Landis ${ }^{1}$ and Vincent Denis ${ }^{2}$ \\ 'NASA John Glenn Research Center, mailstop 302-1, 21000 Brook Park Road, Cleveland, OH 44135 \\ 216-433-2238 e-mail: geoffrey.landis@grc.nasa.gov \\ ${ }^{2}$ International Space University, Parc d'Innovation, 67400 Illkirch-Graffenstaden Strasbourg, France
}

\begin{abstract}
Existing engineering materials allow the constuction of towers to heights of many kilometers. Orbital launch from a high altitude has significant advantages over sea-level launch due to the reduced atmospheric pressure, resulting in lower atmospheric drag on the vehicle and allowing higher rocket engine performance. High-altitude launch sites are particularly advantageous for single-stage to orbit (SSTO) vehicles, where the payload is typically $2 \%$ of the initial launch mass. An earlier paper enumerated some of the advantages of high altitude launch of SSTO vehicles. In this paper, we calculate launch trajectories for a candidate SSTO vehicle, and calculate the advantage of launch at launch altitudes 5 to 25 kilometer altitudes above sea level. The performance increase can be directly translated into increased payload capability to orbit, ranging from 5 to $20 \%$ increase in the mass to orbit. For a candidate vehicle with an initial payload fraction of $2 \%$ of gross lift-off weight, this corresponds to $31 \%$ increase in payload (for 5-km launch altitude) to $122 \%$ additional payload (for $25-\mathrm{km}$ launch altitude).
\end{abstract}

\section{INTRODUCTION}

Existing human-build structures have heights slightly less than one kilometer, however, this height is not limited by materials or construction technology, but rather is limited by the lack of a compelling application for higher towers. Towers of height fifteen to twenty-five kilometers could be easily built using present-day materials. Use of such towers could have great advantages as the launch site of a single stage to robit vehicle.

As an example, table 1 shows the minimum mass required for a tower sized to hold its own weight plus that of a 2000-ton payload at the top of the tower. If the tower material is constructed from a standard construction material, cast steel, the minimum tower mass is approximately two and a half times the weight of the payload at the top. To avoid structural collapse, if made from steel, such a tower would have to be tapered slightly (area taper ratio 2.6:1) from the bottom to top. If a more advanced material with a higher strenght to weight ratio is used, graphite/epoxy composite, the tower is much lower in weight. In this case the required tower mass is only $14 \%$ of the mass of the supported payload, and no taper is needed. Even more advanced materials allow a lower mass yet to be employed. Although these simplified calculated masses do not include nonstructural beams and required auxiliary components, such as (for example) elevators required to lift the vehicle to the top of the tower, cables for bracing, and activedamping control structure for mitigating vibration and wind loads, they serve as a sanity check to show that towers considerably higher than those presently constructed are, in fact, not prohibited by the basic physics of materials.

For extremely high towers, the structure would likely be constructed as a "fractal truss," where the individual beams of a truss are each themselves a truss member, and so forth. An example of such a multi-level truss structure is shown in figure 1.

As discussed by Landis (1998), use of the top of such a tower as the launch site of a rocket would have a long list of advantages. Single stage to orbit (SSTO) vehicles are particularly sensitive to small improvements in launch conditions. Landis (1998) estimated that the payload of a single stage to orbit vehicle could improve by approximately $60 \%$ if the vehicle was launched from fifteen kilometers altitude, instead of launching at sea level.

This is a preprint or reprint of a paper intended for presentation at a conference. Because changes may be made before formal publication, this is made available with the understanding that it will not be cited or reproduced without the permission of the author. 


\section{Structural material: Cast steel: \\ $L c=15.4 \mathrm{~km}$ taper required \\ tower mass 5300 tons \\ (area taper ratio 2.6) \\ Structural material: Graphite epoxy: \\ $L c=107.5 \mathrm{~km}$ No taper needed \\ tower mass 280 tons}

TABLE 1: Example calculations of mass required for a fifteen- $\mathrm{km}$ tower sized to support a 2000 ton launch weight (From Landis 1998), showing the mass required if the tower is made from either cast steel or from a graphite-epoxy composite.

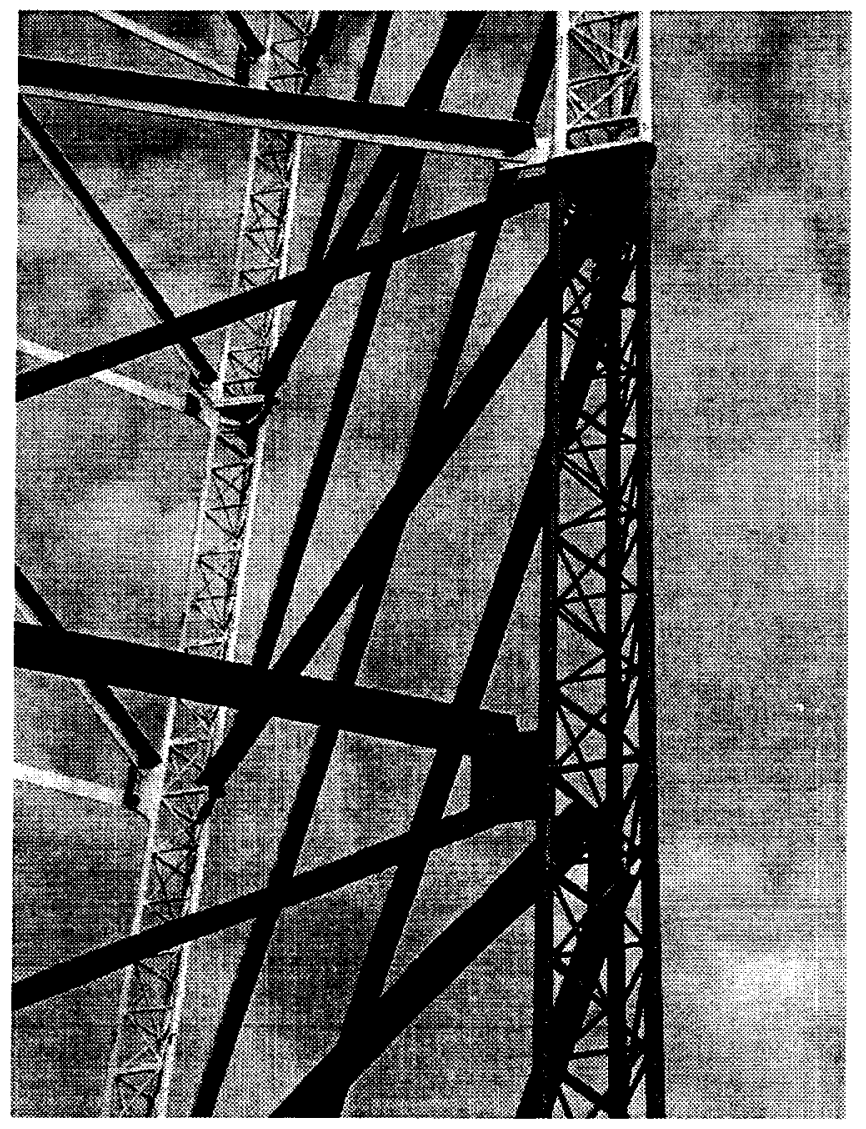

FIGURE 1. Example of a multi-level ("fractal") truss system, where each of the beams of the truss structure is itself a truss

The improvement in performance is primarily due to lower air density. By starting at a lower atmospheric pressure, the vehicle has several design advantages that result in a reduced delta- $V$ required to reach orbit. As well as the reduced drag, the aerodynamic advantages include:

1. Reduced atmospheric drag loss

2. Vehicle can be designed with less attention to aerodynamics.

3. More optimum trajectory curves toward horizontal faster

4. Maximum aerodynamic stress ("Max-Q") occurs at a much lower pressure; lower aerodynamic stress

5. Aerodynamic vibrations lower; allows less robust (lighter) payload 
6. Wind loads on vehicle in flight much lower

7. Acoustic loads much lower

8. Cryogenic storage easier (lower conduction and convective heating)

9. Aero-shroud jettison (for vehicles which jettison non-essential parts) can occur earlier in the trajectory

Figure 2 shows the atmospheric pressure in MPa (equivalent to percentage of one atmosphere) plotted as a function of the launch altitude. Plotted on the same graph is the delta-V required to reach orbit, where the lower aerodynamic drag have been expressed in the form of the required delta- $\mathrm{V}$ to reach orbit. As can be seen from the figure, the velocity increment needed for launching to orbit decreases directly as a function of the initial altitude, and the atmospheric pressure.

In addition, the lower atmospheric pressure means that the rocket operates in an environment which is closer to vacuum. Data on engine performance improvement with the change from near sea-level to near-vacuum conditions has been tabulated by Isakowitz, Hopkins, and Hopkins (1999). For example, the Rocketdyne Atlas MA-5 sustainer engines produce a specific impulse $309 \mathrm{sec}$ operating in vacuum; while the MA-5A booster (essentially the same engine with a nozzle reoptimized for low-altitude operation) produces a specific impulse of 253 seconds at sea level. High altitude operation results in a $22.1 \%$ increase in performance. The increased engine performance can be separated into several components:

1. Higher rocket engine performance at launch due to lower pressure

2. Higher expansion ratio possible

3. Less design compromise needed for operating engine at variable pressure.

4. Lower chamber pressure required to achieve high performance

One of the results of the calculations seen here was that the improvement in engine performance in fact is a significantly larger contribution to the performance improvement than the reduction in atmospheric drag.

In this paper, a trajectory simulation is used to numerically calculate the performance of a candidate SSTO vehicle as a function of the launch altitude.

Finally, launching from an altitude above the weather means no design compromises are needed for weather. These vehicle design advantages are not included in the performance calculations discussed here:

1. Fewer delays for weather

2. Above lightning hazard

3. Lower buffeting due to weather and reduced wind shear means a less robust design needed

\begin{abstract}
APPROACH
In order to evaluate the real benefits of the launch site altitude on rocket performance, a numerical trajectory simulation program was written to compute the final mass as a function of the launch altitude for a single stage to orbit vehicle. The simulation program has been written in Fortran 77, and follows the approach of Sullivan (1990) and Bromley (1998). The launch was assumed to be from a point located on the earth equator, and the final orbit is circular at an altitude of $200 \mathrm{~km}$ (125 miles) Table 2 shows the assumed vehicle parameters

In order to compute the aerodynamic forces, it is necessary to know the coefficients of aerodynamic normal and axial force. These coefficients are function of both Mach number and angle of attack. Aerodynamic forces were computed using a set of equations interpolated from the aerodynamic characteristics quoted by Sutton (2001).

The engine performance varies as a function of external pressure. The performance was calculated at each point in the trajectory, and the average specific impulse (Isp) was then calculated by dividing the total impulse produced over the flight by the total fuel consumed.
\end{abstract}




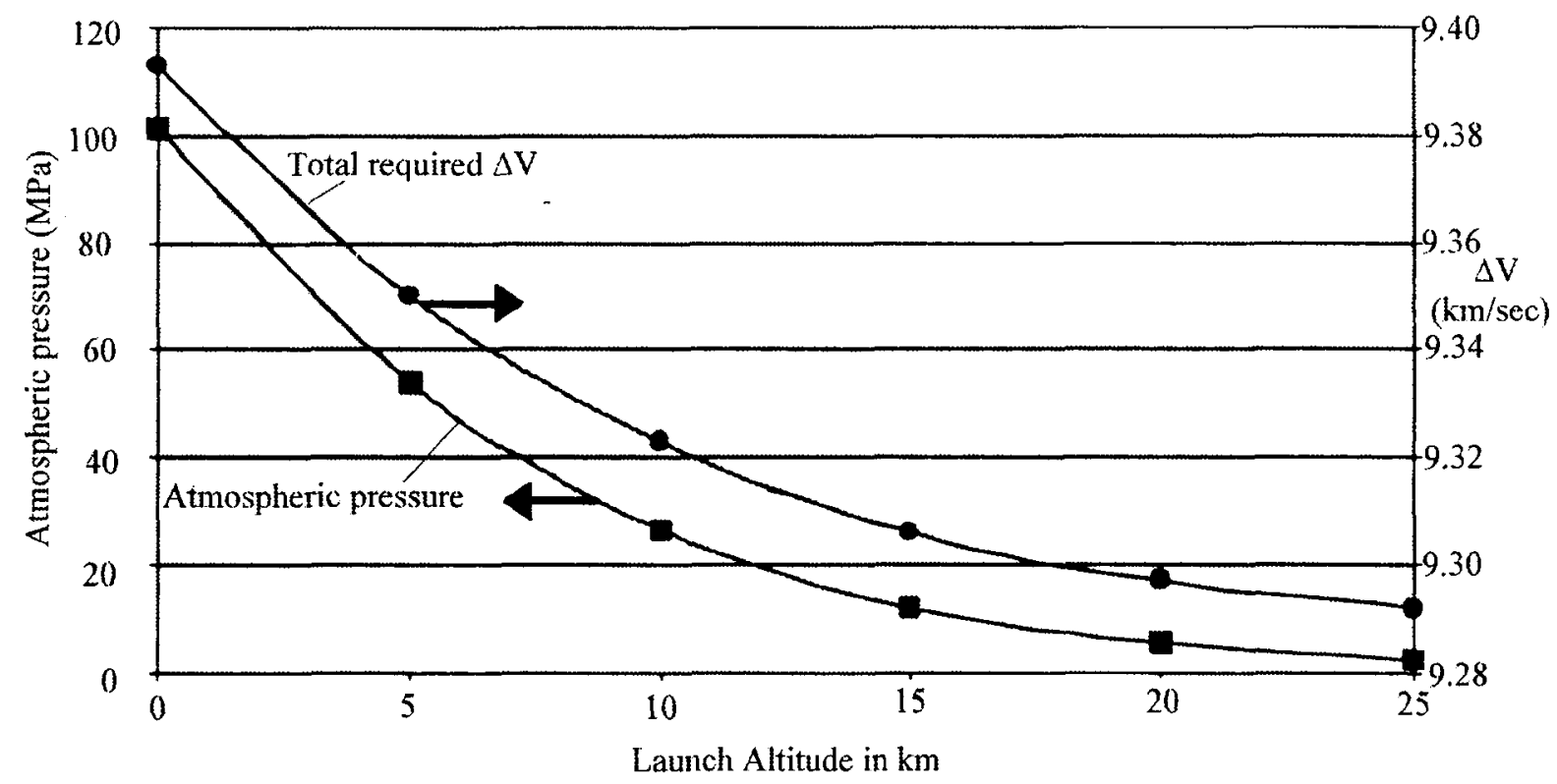

FIGURE 2. Atmospheric pressure and delta- $V$ required to reach orbit are plotted as a function of the initial launch altitude for a candidate SSTO vehicle.

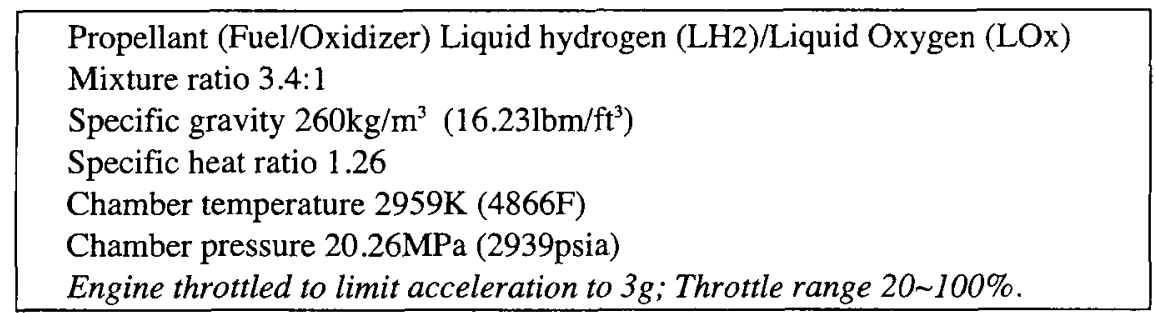

TABLE 2: Parameters of the example SSTO vehicle used in the trajectory simulation

\section{RESULTS}

Figure 2 shows the atmospheric pressure in $\mathrm{MPa}$ (equivalent to percentage of one atmosphere) plotted as a function of the launch altitude. Plotted on the same graph is the delta- $V$ required to reach orbit. As can be seen from the figure, the velocity increment needed for launching to orbit decreases directly as a function of the initial altitude, and the atmospheric pressure.

Figure 3 plots the results of the trajectory simulation. The total propellant mass is plotted as a fraction of the initial mass ("Gross Lift-off Mass"). The required propellant loading for the example SSTO vehicle decreases with altitude from $87.5 \%$ of the gross lift-off mass, down to slightly over $85 \%$ of the gross lift-off mass for a launch altitude of $25 \mathrm{~km}$ above sea level.

Since for the candidate SSTO the payload is typically on the order of $2 \%$ of the gross lift-off mass, this decrease in required fuel mass potentially represents a large increase in payload. The performance increase can be directly translated into increased payload capability to orbit, ranging from $5 \%$ increase in mass to orbit for a 5-kilometer launch altitude, to a $19.68 \%$ increase in the mass to orbit for a $25-\mathrm{km}$ launch altitude. For a candidate vehicle with an initial payload fraction of $2 \%$ of gross lift-off weight, this corresponds to $31 \%$ increase in payload (for $5 \mathrm{~km}$ launch altitude) to $122 \%$ additional payload (for $25 \mathrm{~km}$ launch altitude). 
Table 3 breaks down the improvement in performance into two components. The effect of decreased drag due to lower atmospheric pressure is seen as a decrease in the delta- $\mathrm{V}$ required to reach orbit. The improvement in the specific impulse of the engine is averaged over the flight, and expressed as the improved Isp. As can be seen, the majority of the performance improvement is due to the increased specific impulse achieved by operating the rocket engines at a lower ambient pressure.

\begin{tabular}{|c|c|c|c|c|}
\hline $\begin{array}{c}\text { Launch } \\
\text { Altitude } \\
(\mathrm{km})\end{array}$ & $\begin{array}{c}\text { Decrease in drag } \\
\text { (expressed as \% reduction } \\
\text { in required Delta-V) }\end{array}$ & $\begin{array}{c}\text { Improvement } \\
\text { in average Isp } \\
(\%)\end{array}$ & $\begin{array}{c}\text { Increase in final } \\
\text { mass to orbit } \\
(\%)\end{array}$ & $\begin{array}{c}\text { Increase in } \\
\text { payload } \\
(\%)\end{array}$ \\
\hline 0 & - & - & 0 & 0 \\
\hline 5 & $0.30 \%$ & $4.70 \%$ & $5.00 \%$ & $31.2 \%$ \\
\hline 10 & $0.94 \%$ & $8.70 \%$ & $9.64 \%$ & $60.2 \%$ \\
\hline 15 & $1.24 \%$ & $12.25 \%$ & $13.49 \%$ & $84.3 \%$ \\
\hline 20 & $1.39 \%$ & 15.40 & $16.79 \%$ & $104.9 \%$ \\
\hline 25 & $1.46 \%$ & $18.22 \%$ & $19.68 \%$ & $122.5 \%$ \\
\hline
\end{tabular}

TABLE 3: Calculated contributions of drag reduction and engine performance increase on launch vehicle performances, compared to sea level launch. The final columm, increase in payload, is calculated assuming that the payload mass is $2 \%$ of the gross lift-off mass for the sea-level launch.

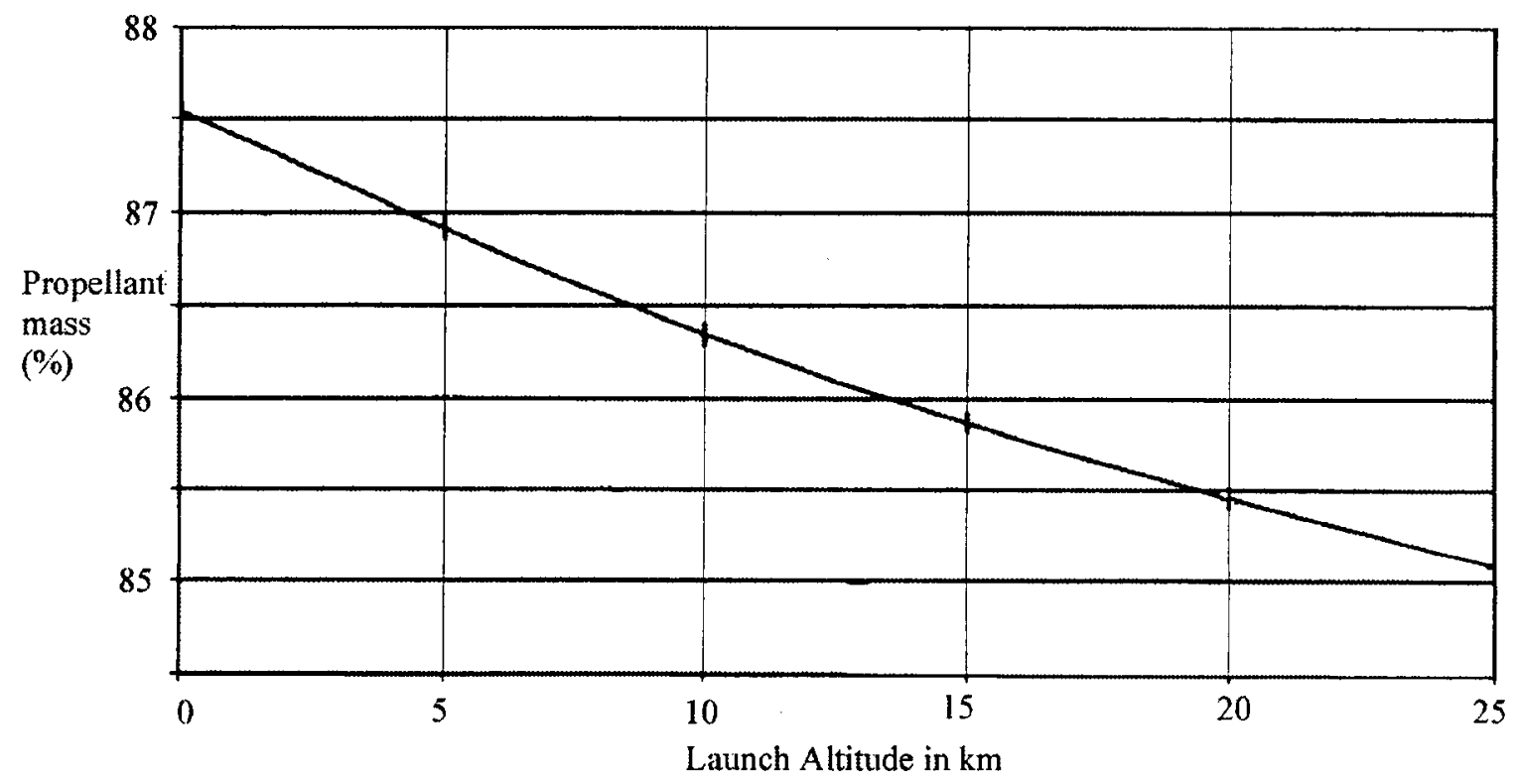

FIGURE 3. The required propellant mass to reach orbit for a candidate SSTO vehicle, expressed as a percentage of the initial vehicle gross lift-off mass, is plotted as a function of the initial launch altitude.

\section{CONCLUSIONS}

Towers of height fifteen to twenty-five kilometers could be easily built using present-day materials. Launch from the top of such a tower would have a long list of advantages. The improvement is primarily due to lower air density.

The results of the trajectory simulation show that the required propellant loading for a candidate SSTO vehicle decreases as launch altitude is increased,. The propellant launch fraction is $87.5 \%$ of the gross lift-off mass for a sea-level launch, and decreases to slightly over $85 \%$ of the gross lift-off mass for a launch altitude of $25 \mathrm{~km}$ above 
sea level. Since for the candidate SSTO the payload is typically on the order of $2 \%$ of the gross lift-off mass, this decrease in required fuel mass can result in over a factor of two increase of payload mass. Even a five kilometer launch altitude, equivalent to launching from the top of a moderate terrestrial mountain, would result in a significant improvement in payload.

It is very likely that other advantages of tower launching which were not analyzed could contribute significantly larger increases in payload, as well as operational simplicity. For example, the possible benefits of not having to compromise the design to deal with aerodynamic loads at sea-level could be far more significant than the performance gains analyzed. Such a tower launch would also be advantageous for advanced launch technologies, such as mass-driver, laser, microwave, or ram-accelerator launch.

Higher towers have also been proposed; for example, Landis and Cafarelli (1995) examined the use of towers of up to $2250-\mathrm{km}$ altitude, extending well outside the Earth's atmosphere, and concluded that they might be feasible with advanced materials. Such a tower could be one component of a geosynchronous tower, or "space elevator." A 15$\mathrm{km}$ tower could be a technology demonstration and a stepping-stone to more ambitious tower systems.

\section{ACKNOWLEDGEMENTS}

The work of Vincent Denis at the NASA Glenn Research Center was sponsored by the International Space University Master's in Space Studies 2000/2001 program, with Tarik Kaya as the advisor.

\section{REFERENCES}

Bromley, B. P., Ballistic trajectory mission analysis for a single stage to orbit re-usable launch vehicle using nuclear thermal rocket engines and hydrogen propellant, NE-411, final project report, University of Illinois at Urbana-Champaign, April 1998

Isakowitz, S. J., Hopkins, J. P. and Hopkins, J. B.: International reference guide to space Launch systems, 3rd ed. American Institute of Aeronautics and Astronautics, Inc., Reston Virginia, 1999

Landis, G. A., "Compression Structures for Earth Launch," paper AIAA-98-3737, 24th AIAA/ASME/SAE/ASEE Joint Propulsion Conf., July 13-15, 1998, Cleveland OH. In earlier form: 7th Advanced Space Propulsion Workshop, Jet Propulsion Laboratory, 9-11 April 1996.

Landis, G. A., and Cafarelli, C., "The Tsiolkovski Tower Reexamined," J. British Interplanetary Society, Vol. 52, 175-180 (1999). Presented as paper IAF-95-V.4.07, 46th International Astronautics Federation Congress, Oslo Norway, 2-6 Oct. 1995.

Sullivan J. M.: Trajectory optimisation for an asymmetric launch vehicle, Master of Science Thesis, Massachusetts Institute of Technology, June 1990.

Sutton, G. P. and Biblarz, O.: Rocket Propulsion Elements, 7th ed. Jonn Wiley \& Sons, Inc., New York, Seventh edition (2001). 